TH. PöschL: Einführung in die analytische $M e-$ chanik. Veriag G. Braun, Karlsruhe 1949. VIII und $166 \mathrm{~S}$. mit $37 \mathrm{Abb}$. HIn. DM 10,-.

Diese Einführung gründet sich auf Vorlesungen, die der Verfasser während der letzten Jahre an der Technischen Hochschule in Karlsruhe gehalten hat, und legt Zeugnis ab für die gediegene, auf A. ClebsCE und H. Hertz zurückreichende Karlsruher Tradition. Ihr Erscheinen kommt einem fühlbaren Mangel nach einem kurz gefaßten und zuverlässigen Lehrbuche der analytischen Mechanik sehr entgegen. Das Buch ist zur Vorbereitung des Studiums größerer Spezialwerke und von Originalabhandlungen bestens geeignet.

Nach Formulierung der Aufgabenstellung und einem kurzen geschiehtlichen Überblick folgt die Dynamik der Punktmassen und starren Körper (85 S.). Hierfür werden die wichtigsten elementaren Methoden, die in der allgemeinen Mechanik grundlegende Bedeutung erlangt haben, zusammengestellt: LAGRaNGesche Bewegungsgleichungen, Energieintegral, zyklische Koordinaten, D'Aiembertsches Prinzip, Eulersche Gleichungen, Differentiaiprinzipe von
Gauss und Hertz. Zwei weitere Kapitel sind den Hauptsätzen der Variationsrechnung als Grundlagen der Integralprinzipien (36 S.) und der Anwendung dieser Prinzipien auf die Dynamik (40 S.) gewidmet. Sie enthalten die Hamilton-Jacobische Theorie und die von Hilbert und seinen Schülern erzielten Fortschritte der Variationsrechnung für die Integrations- und Transformationstheorie. Mit Hilfe der kanonischen Aquivalenz wird der Zusammenhang zwischen der klassischen analytischen Mechanik und der älteren Quantentheorie hergestellt.

Alle theoretischen Ausführungen sind streng und werden durch 24 gut gewählte Beispiele, die vorwiegend das Zweikörperproblem, das räumliche Pendel, die Kreiselbewegung and die geodätischen Linien auf Rotationsflächen und dem dreiachsigen Ellipsoid zum Gegenstand haben, wirkungsvoll ergänzt. - Ein Auszug der Lehrbuchliteratur ist am Ende des Buches angefügt; wünschenswert wären auch genaue Zitate von gelegentlich im Text herangezogenen Originalarbeiten.

H. BiLHARz (Freiburg-Oberwolfach)

\title{
Zuschrift an die Schriftleitung
}

\section{Hermann Schmid, Braunschweig: Bemerkung zu einem Determinantensatz.}

In seiner Note ,Verallgemeinerung eines Interpolationsproblems“ (dieses Archiv 1 (1948/49, 278-281) berechnet Herr WENDELIN den Wert derjenigen Determinante, die aus

$$
\left|\begin{array}{ccccc}
x^{n} & x^{n-1} & \ldots & x & 1 \\
x_{\nu}^{n} & x_{v}^{n-1} & \ldots & x_{\nu} & 1
\end{array}\right| \nu=1,2, \ldots \ldots n
$$

durch Streichen der ersten Zeile und $\operatorname{der}(n-i+1)$-ten Spalte hervorgeht. $(n>1, i=0,1, \ldots n$.) Es sei mir der Hinweis gestattet, daß das Ergebnis nicht neu ist; es findet sich z. B. bei PolyASzEGö, Aufgaben nnd Lehrsätze aus der Analysis, 2. Bd., Berlin 1925, S. 99, Aufg. 10. Besonders einfach ergibt es sich übrigens daraus, daß (1) als VANDERMondesche Determinante der $n+1 \mathrm{Un}$ bestimmten $x, x_{1}, x_{2}, \ldots x_{n}$ den Wert hat

$$
\left(x-x_{1}\right)\left(x-x_{2}\right) \ldots\left(x-x_{n}\right) \prod_{1 \leq \mu<\nu \leq n}\left(x_{u}-x_{\nu}\right)
$$

durch Gleichsetzung der Faktoren von $x^{i}$ in beiden Darstellungen. (Eingegangen am 19. 4. 1949. Die Zuschrift hat Herr H. WeNDELIN, Graz, dankend zur Kenntnis genommen. Er wünscht darauf hinzuweisen, daß der Satz selbst von obiger Bemerkung nicht betroffen wird. Die Schr.)

(Abgeschlossen am 31. Mai 1949) 